\title{
DNA vaccines expressing conserved elements provide potent and broad immune responses
}

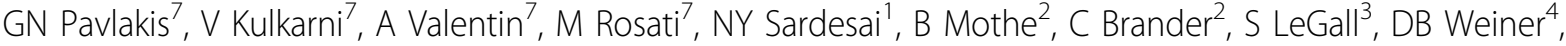 \\ M Rolland $^{5}, \mathrm{Jl}_{\text {Mullins }}{ }^{6}$, BK Felber $^{7 *}$
}

From AIDS Vaccine 2012

Boston, MA, USA. 9-12 September 2012

\section{Background}

Immunodominance and sequence diversity are major hurdles in the development of effective HIV vaccines. We tested the hypothesis that a vaccine candidate composed of strictly Conserved Elements (CE) of the HIV proteome excluding the variable regions would help overcome problems of viral sequence diversity and potential negative effects of immunodominance. Seven $\mathrm{CE}$ were identified in $\mathrm{p} 24^{\mathrm{gag}}$. Vaccination of macaques with $\mathrm{p} 55^{\mathrm{gag}} \mathrm{DNA}$ failed to elicit cellular or humoral immune responses to the $\mathrm{CE}$, while epitopes outside of the $\mathrm{CE}$ were immunogenic.

\section{Methods}

Two HIV p24 ${ }^{\mathrm{gag}} \mathrm{CE}$ DNA plasmids were generated, providing potential epitopes found in $>99 \%$ of all HIV-1 M group sequences. DNA vectors, optimized for gene expression were used to immunize mice and macaques by IM injection followed by in vivo electroporation.

\section{Results}

Vaccination with p24 ${ }^{\mathrm{gag}} \mathrm{CEvac}$ DNAs elicited potent, cross-clade cellular and humoral immune responses. Highly cytotoxic CE-specific T cells, capable of Granzyme $\mathrm{B}$ production and degranulation, were generated. Importantly, boosting of the CEvac-primed macaques with $p 55^{\mathrm{gag}} \mathrm{DNA}$ greatly augmented the CE-specific cellular responses (up to 10-fold) as well as humoral responses, despite the failure of $\mathrm{p} 55^{\mathrm{gag}} \mathrm{DNA}$ vaccine to induce de novo CE-specific responses. CEvac DNA prime-p55 ${ }^{\mathrm{gag}} \mathrm{DNA}$ boost in mice led to similar conclusions. Interestingly, mapping analysis showed differential increase of the CE-specific responses by the p $55^{\mathrm{gag}} \mathrm{DNA}$ boost, demonstrating changed hierarchy of CE responses in macaques.

\section{Conclusion}

Vaccination with the $\mathrm{p} 24^{\mathrm{gag}} \mathrm{CEvac}$ DNA overcame the problem of diversity by generating strong cross-clade Gag-specific immune responses, and of immunodominance, eliciting responses to subdominant but highly conserved elements, and also by broadening the p5 $5^{\mathrm{gag}}$ DNA induced immunity. $\mathrm{p} 55^{\mathrm{gag}}$ DNA did not induce de novo responses to the $\mathrm{CE}$, but was able to significantly boost pre-existing CE-induced responses and alter the hierarchy of these responses. Translation of this concept into clinical trials may elicit cross-clade cellular immune responses against components of the viral proteome with limited capacity for immunological escape.

\section{Author details}

'Inovio Pharmaceuticals, Inc., Blue Bell, PA, USA. ${ }^{2}$ IrsiCaixa-HIVACAT, Barcelona, Spain. ${ }^{3}$ Ragon Institute of MGH, MIT and Harvard, Boston, MA, USA. ${ }^{4}$ University of Pennsylvania, Philadelphia, MD, USA. ${ }^{5}$ U.S. Military HIV Research Program, Rockville, MD, USA. ${ }^{6}$ University of Washington, Seattle, WA, USA. ${ }^{7}$ Frederick National Laboratory for Cancer Research, Frederick, MD, USA.

Published: 13 September 2012

doi:10.1186/1742-4690-9-S2-067

Cite this article as: Pavlakis et al:: DNA vaccines expressing conserved elements provide potent and broad immune responses. Retrovirology 2012 9(Suppl 2):067. 